\title{
Auto adjust masses of automotive structures with desired centre of gravity
}

\begin{abstract}
In this study a subroutine was developed and added in the pre-processing module of the frame work of commercial package of ANSYS to distribute the extra masses according to specific assigned centre of gravity (COG). The work further calculate the first bending and torsional natural frequencies of the simplified body in white (BIW) model of automotive in order to maximize these frequencies with final mass constraint. It is found that adding the extra masses symmetrically about longitudinal axis helps to improve the first bending and torsion natural frequencies. However, removing the mass along this axis leads to have higher values in the case of first bending and natural frequencies.
\end{abstract}

Keyword: ANSYS; Centre of gravity; Finite element; Mass distribution; Tubular structure 